\title{
Coupled FEM-BEM crack growth analysis
}

\author{
L. Zhang \& R. A. Adey \\ Wessex Institute of Technology, Ashurst Lodge, Southampton, UK
}

\begin{abstract}
The increasing interest in large structure fracture analysis has heightened the need for efficient numerical computational tools suitable to predict crack propagation. While a sub modelling approach can be used in some cases it does not take into account the redistribution of the loads in the structure thus requiring a large part of the structure to be included in the crack growth model.

Generally large structures are modelled with finite element methods (FEM) because of the many varied types of structural element. Modelling crack growth with FEM results in a particularly complex remeshing process as the crack propagates. Hence, self-adaptive remeshing is one of the major features that must be incorporated in the construction of a computational tool to properly perform crack propagation analysis with the FE method.

The boundary element method (BEM) has attracted lots of attention in the field of fracture mechanics as it simplifies the meshing process and has the ability to accurately represent the singular stress fields near the crack front. One challenge is how the two methods can work together efficiently for a large structure. A new FEM-BEM method is therefore proposed to perform such crack growth analyses.

This paper describes the methodology of a coupled FEM-BEM crack growth analysis for a large scale structure. Both finite element software ABAQUS and boundary element software BEASY were used in the analysis. Several examples are presented at the end of the paper including crack growth in a gear tooth and in a stiffened panel respectively.
\end{abstract}

Keywords: finite element, boundary element, coupling method, fracture mechanics, crack growth, stress intensity factor. 


\section{Introduction}

Fracture mechanics represents the applied mechanics framework necessary for the description of the behaviour of cracked components under applied loads. It can be used to predict how cracks will affect the durability and life of components and structure. The finite element method is employed in many engineering areas including fracture mechanics. Many methods have been developed to solve fracture problems during the last twenty years [1]. However, for crack growth problems, the continuous remeshing process has been a difficulty for most finite element methods [2]. Dual Boundary element, on the other hand, is more flexible as only the boundary needs to be discretized during the analysis [3-6]. The purpose of the current research is to minimize the extent of the remeshing process, yield as accurate results as possible and enable crack propagation to be simulated in large scale structures with different element types. An automatic FE crack propagation code was developed and based on this an FE-BE sub-modelling code has been developed to perform automatic crack propagation analysis. The FE method will be used to compare the results with coupled FE-BE method and the latter incorporates the capabilities of both BEASY10.0 and ABAQUS 6.5. This paper focuses on Mode I and II crack propagation. We will show some examples of edge crack propagation in $2 \mathrm{D}$ structures and edge crack propagation in 3D thin structures to illustrate the applications of the proposed method.

\section{Methodology of the coupled FE-BE crack growth method}

The finite element method is a robust method for elastic and nonlinear material problems. There are numerous pre-processing programmes capable of translating CAD models into finite element models such as PATRAN, GID, etc. The boundary element method can model cracks without remeshing the domain, which significantly simplifies the analysing process. Using coupled FE-BE automatic method allows us to employ advantages of both methods in fracture mechanics problems, especially for the models in which cracks only exist in local areas. In the proposed coupled FE-BE method, instead of applying direct coupling of the $\mathrm{BE}$ and $\mathrm{FE}$ solution matrices by either presenting the $\mathrm{BE}$ matrices as stiffness matrices or transforming the FE forces into tractions and linking them with the tractions in the BE matrices, two models are created, the original FE model and a local $\mathrm{BE}$ model representing the crack. The displacements or hybrid displacement-traction values calculated from the $\mathrm{FE}$ model are used as prescribed boundary conditions for the local BE model [3]. FE stress values, however, are not used since in finite element analysis stresses are obtained by differentiating displacements, in which process computational errors might be introduced. As the stresses and the displacements change during the crack propagation process, the boundary conditions on the local model must be updated in order to take into account the redistribution of stresses as the crack grows. In the proposed approach changes in strain energy is used as a criterion to 
determine whether the boundary conditions of the sub-model need updating. This will be explained in more detail in chapter 4 . The following is a flowchart of the proposed coupled FE-BE method:

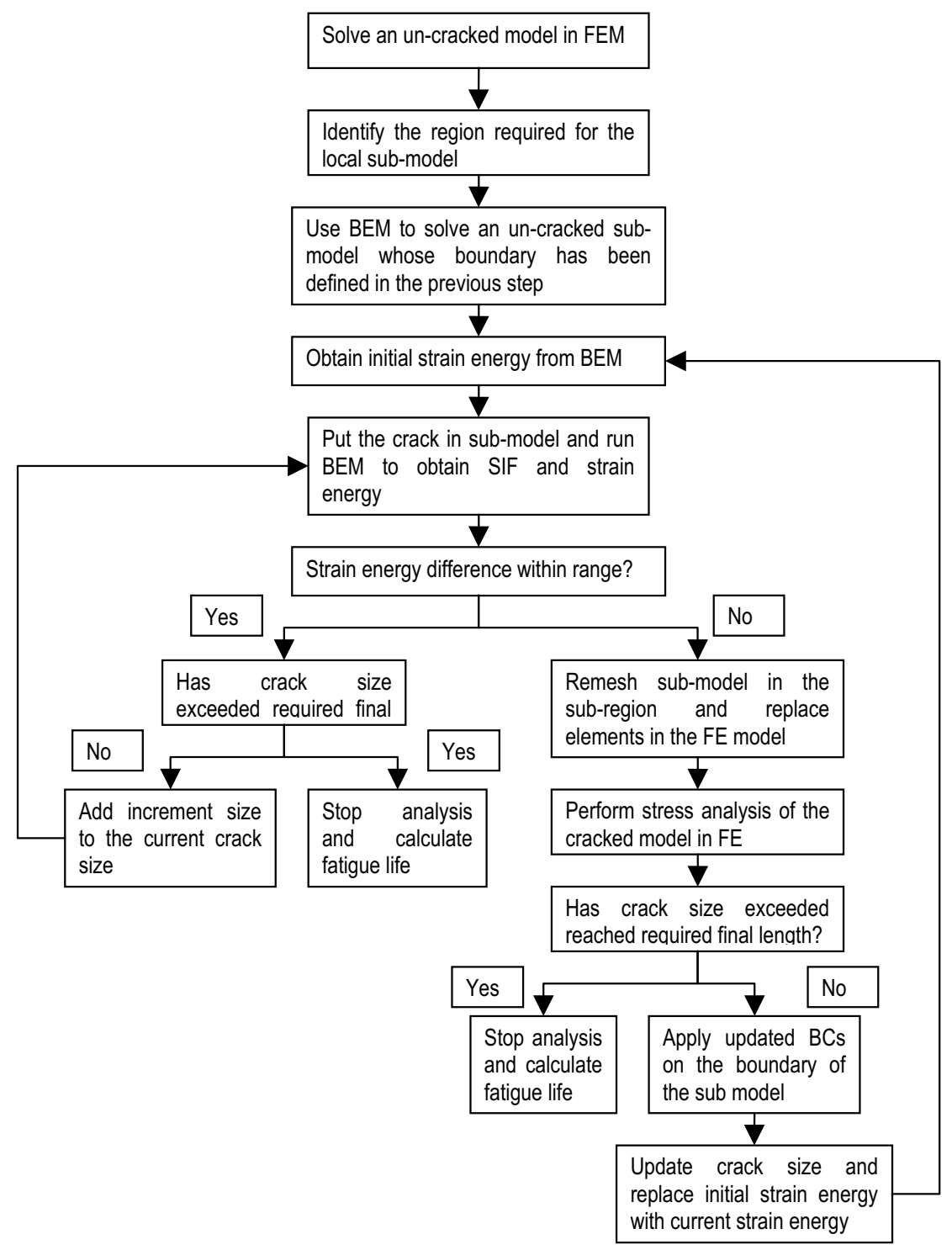

Figure 1: $\quad$ Flowchart of the directly coupled FE-BE crack growth method. 


\section{BEM sub-model generation and local remeshing}

A region is first created to identify the sub-model boundary (Figure 2). The length of the edge can be expressed in the following equation:

$$
L_{S}=L_{\text {frack }}+D_{\text {gap }}
$$

where $L_{S}$ represents the side length of the square, $L_{f C r a c k}$ is the length of the final crack size and $D_{g a p}$ defines the region as measured form the crack that is to be included in the sub model. The second term on the right hand side of the equation can be approximated by iteratively using the following formula:

$$
D=\frac{l_{1}-q l_{n}}{1-q}
$$

where $D$ is the gap distance, $l_{1}$ is the crack increment size and $l_{n}$ is the side length of the elements that intersect the circle and $q$ is the aspect ratio of the gap mesh. In general, aspect ratios should be less than 2.5:1 in order to avoid large calculation errors. Take a flat-plate under tension for example. Assuming the initial crack size is $2 \mathrm{~mm}$ and the estimated final crack size is $10 \mathrm{~mm}$, so $L_{f \text { Crack }}$ equals $10 \mathrm{~mm}$. After iteration, if the minimum gap found is $5 \mathrm{~mm}$, then the final side length of the square is $10+5=15 \mathrm{~mm}$.

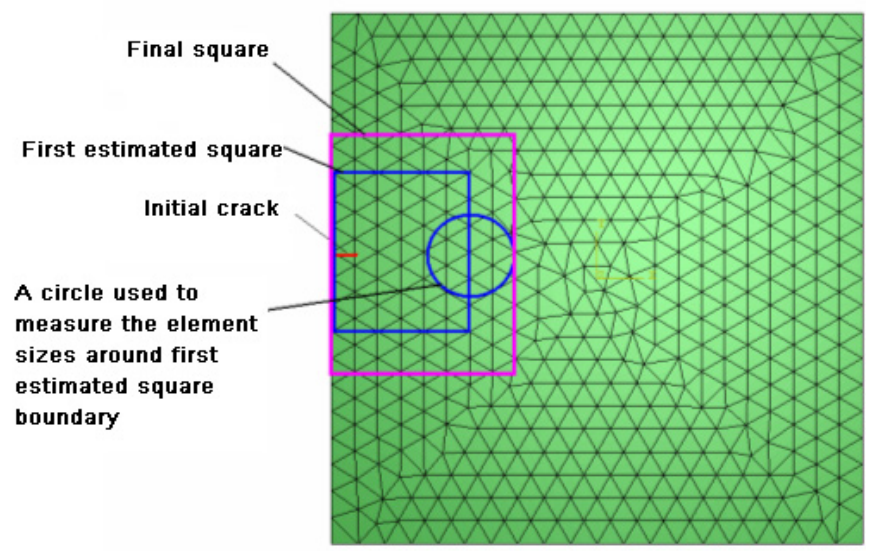

Figure 2: Determination of a square used to generate the sub-model boundary.

Once the sub-model boundary has been determined, the next step to insert the initial crack in the model and perform a fatigue crack growth analysis. The crack type chosen for the analysis is a straight line, 2-element crack. Before putting the crack inside the sub-model boundary, it is necessary to compare the element size on the initial crack and the size of the elements connecting the initial crack to the boundary. If the original element size is too big, it must be divided into smaller pieces and map them onto the original curve. 
Boundary Elements and Other Mesh Reduction Methods XXIX 271
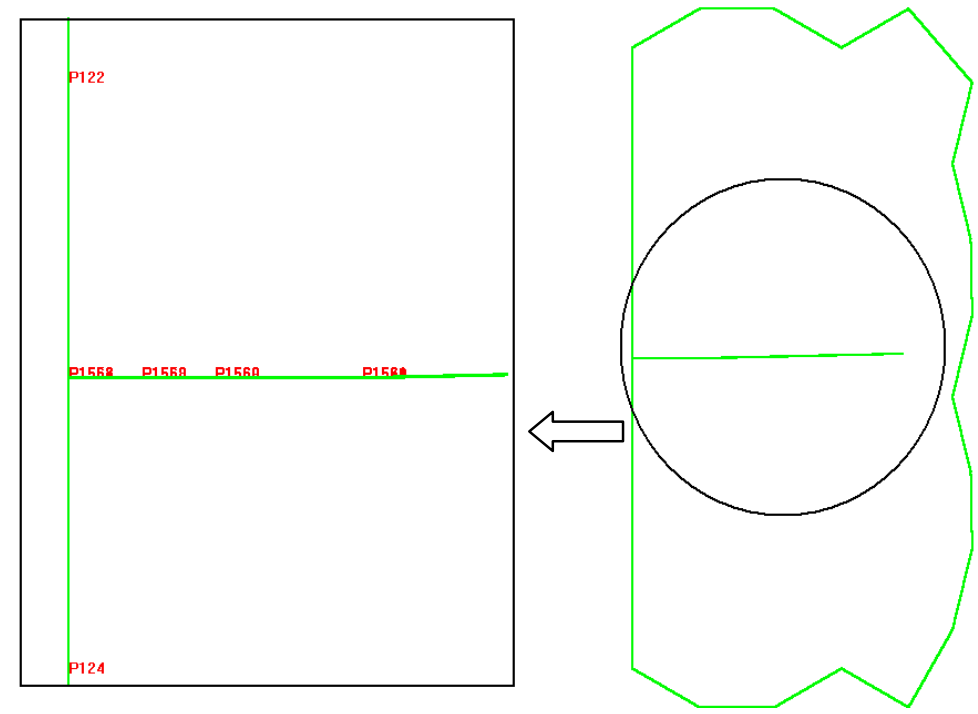

Figure 3: A focused view of a BEM sub-model abstracted from: a plate.

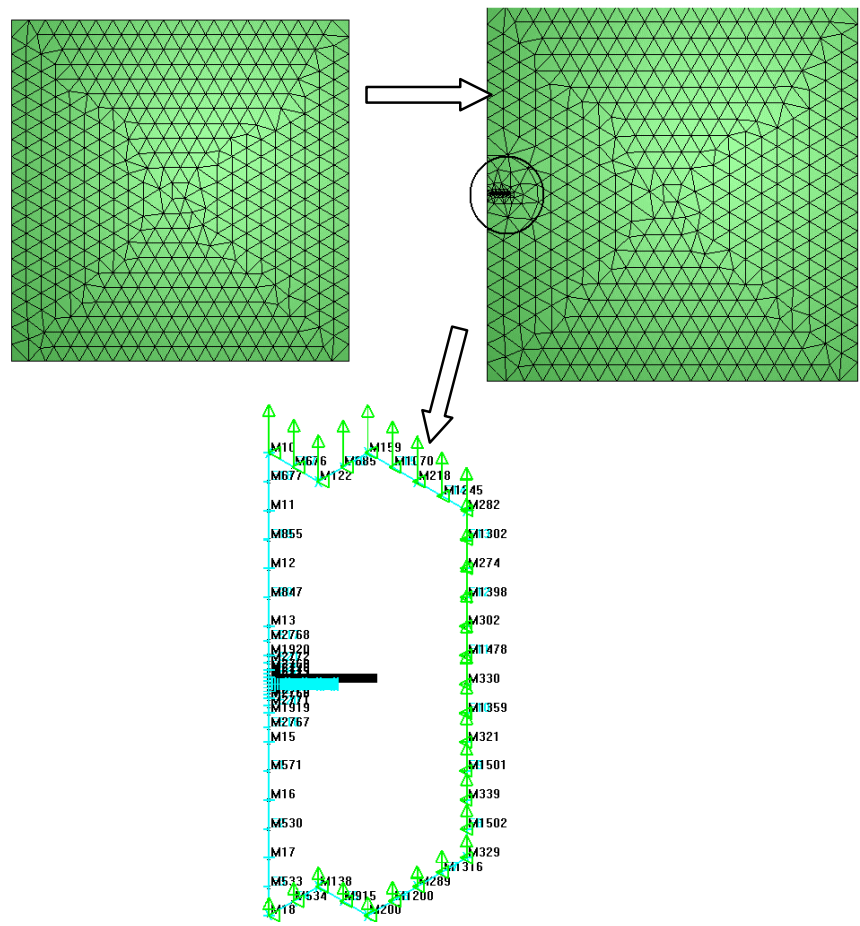

Figure 4: $\quad$ Boundary condition abstracting process. 


\section{Updating boundary conditions and re-analysis in FEM}

The crack propagation in the local sub model continues until the change in the strain energy is larger than the specified criteria. At this point the sub-model including the crack is remeshed as a FE model so it can be added back into the original FE model. Because this new model will not be used to calculate any fracture mechanics data it is not necessary to use any special crack elements or use a refined grid near the crack. The mesh has simply to be sufficient to model the general stress distribution near the crack but not at the crack tip. The whole model is then re-analysed to provide a new solution which can be used to identify the new boundary conditions for the sub-model, which is illustrated in Figure 4.

\section{Examples}

\subsection{Example-edge crack at the root of a gear tooth}

This test will investigate crack propagation in a mixed mode crack growth situation. The test results will be compared with those obtained from the boundary element method.

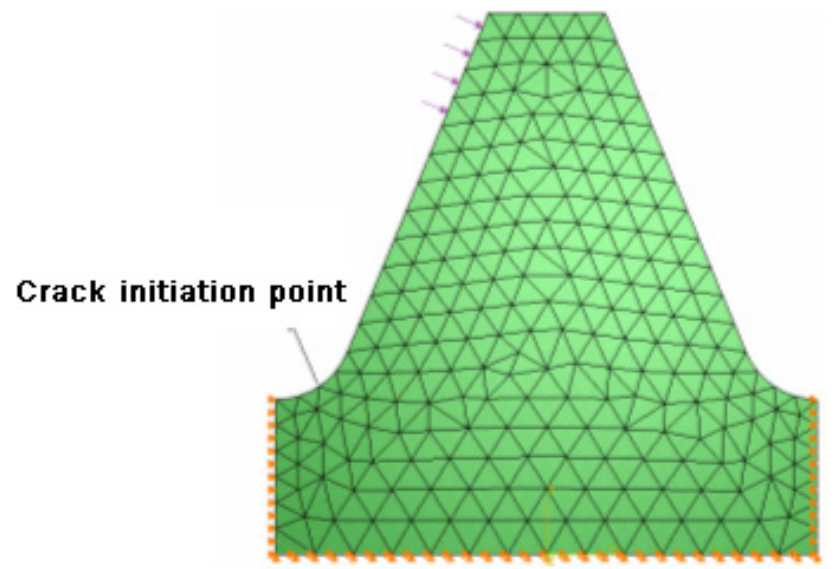

Figure 5: A gear tooth under contact pressure during engagement.

\subsubsection{Input parameters}

a) Geometry definitions:

Height of the tooth: $14 \mathrm{~mm}$, Width of the tooth: $3 \mathrm{~mm}$ (top); $14 \mathrm{~mm}$ (bottom), Fillet radius at the root of the gear tooth: $2 \mathrm{~mm}$.

b) Parameters in automatic coupled FE-BE method:

Geometry: as shown in Figure 5. 
Boundary conditions: $1500 \mathrm{MPa}$ pressure applied on one side the tooth to simulate the engagement of the gear tooth, Crack initiation angle: 315 degrees, Initial crack length $=0.1 \mathrm{~mm}$, Number of elements on initial crack: 2 for AutoFEBE, Number of steps: 30, Increment size: $0.1 \mathrm{~mm}$, Element type: 6-node quadratic plane strain triangle (CPE6) [7], Re-analysis criterion: Strain energy difference $>=2 \%$

\subsubsection{Results}

Figure 6 shows a comparison of the stress intensity factor vs crack size for the three different methods. As can be seen there is close agreements for the SIF and the crack path as shown in Figure 7. The FE mesh required in the FEBE approach is clearly much less refined than that required when the finite elements are used to approximate the stress field close to the crack.

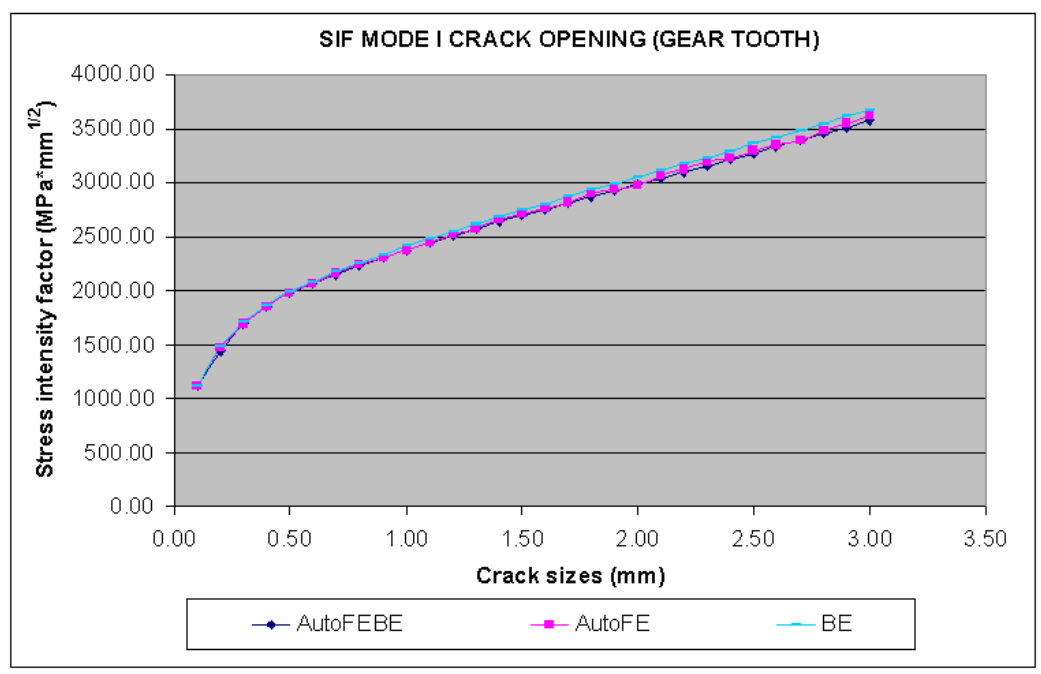

Figure 6: $\quad$ Stress intensity factor vs. crack size for mode I crack opening.
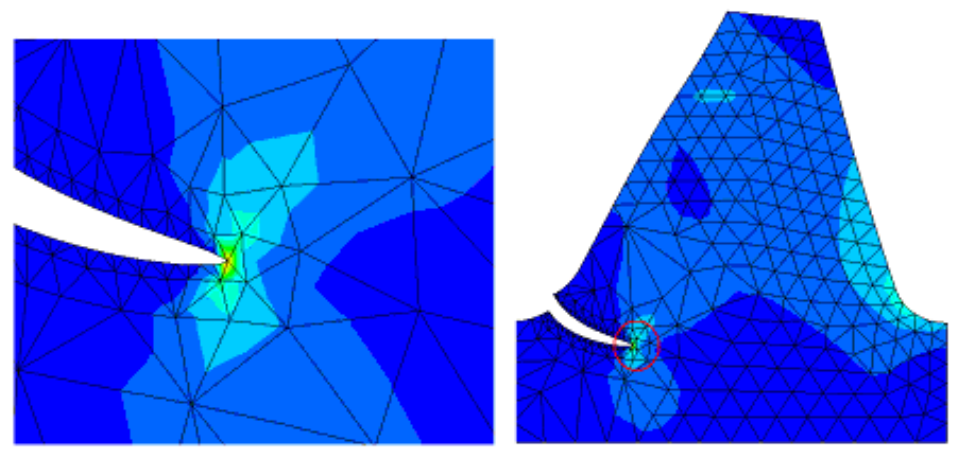

Figure 7: Deformed gear tooth in coupled FEBE method. 


\subsection{Example-stiffened panel with an imbedded crack in the centre}

\subsubsection{Test set-up}

The two stringer specimens (2SP), Figure 8, with a sheet and outer flange thicknesses of 2.5 and $0.5 \mathrm{~mm}$ respectively, and a stringer height of $25 \mathrm{~mm}$, were tested on a servo-hydraulic machine by Llopart et al [8]. The applied load ratio (R) was 0.1 with a maximum load of $60.3 \mathrm{KN}$. The initial crack length was $2 \mathrm{a}_{0}=3 \mathrm{~mm}$. An anti-bending device was used in the original tests to ensure only mode I crack opening exists.

\subsubsection{Model simplification}

As the model is symmetric with regard to the geometrical centre, it can be simplified as a plate with an edge crack in the middle. In addition, as the plate is very thin in comparison to its width and length, the whole structure is modelled with shell elements in FE. Since only the stress distribution around the crack is important, some simplifications have been made in order to reduce computational time. For example, the holes on both ends and the fillets between the stiffener and the panel are ignored. The load is directly applied on the edge of the plate while the plate is fixed in normal direction to prevent bending.

\subsubsection{Input parameters}

Element type: quadratic triangular shell elements STRI65 [7]

Initial crack size: $2 \mathrm{a}_{0}=10 \mathrm{~mm}$

Number of increments: 5

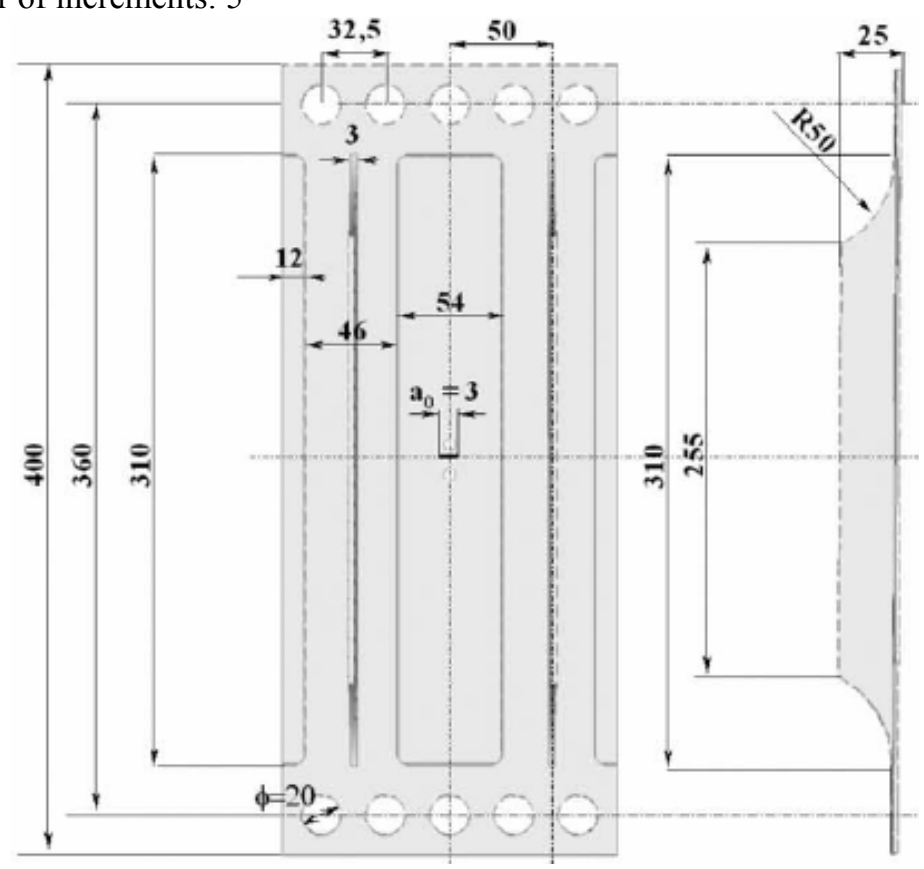

Figure 8: $\quad$ A two stringer specimen [9]. 


\subsubsection{Results}

Figure 9 shows the stress distribution and deformation of the stiffened panel after crack propagation. A comparison has been made between the numerical results from the proposed method and the test results from literature [8]. A good agreement is found between the two methods, which can be observed from Figure 10.

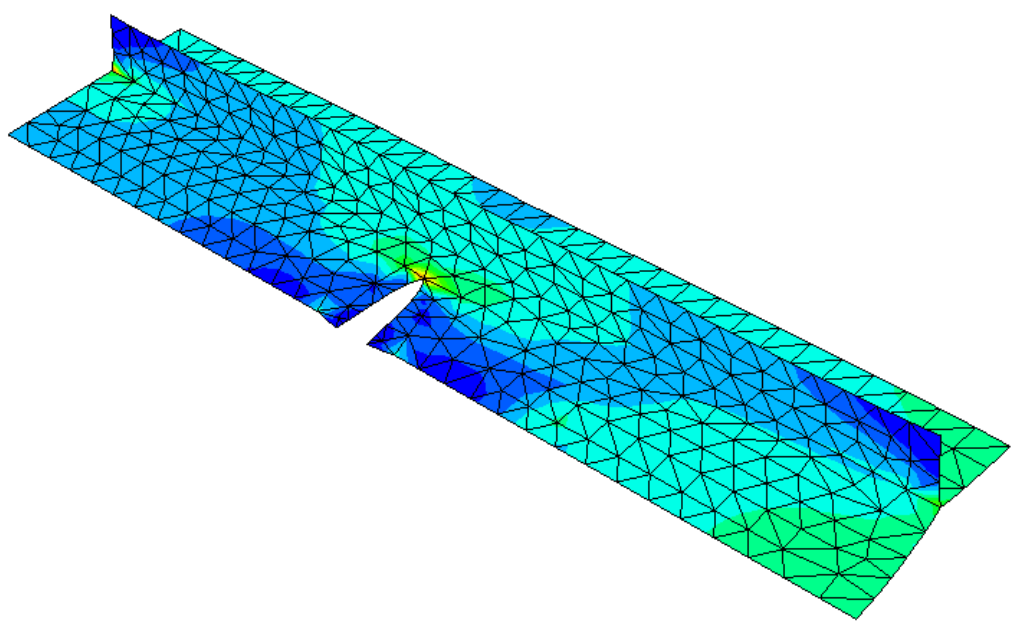

Figure 9: Stress distribution of the stiffened panel after crack propagation.

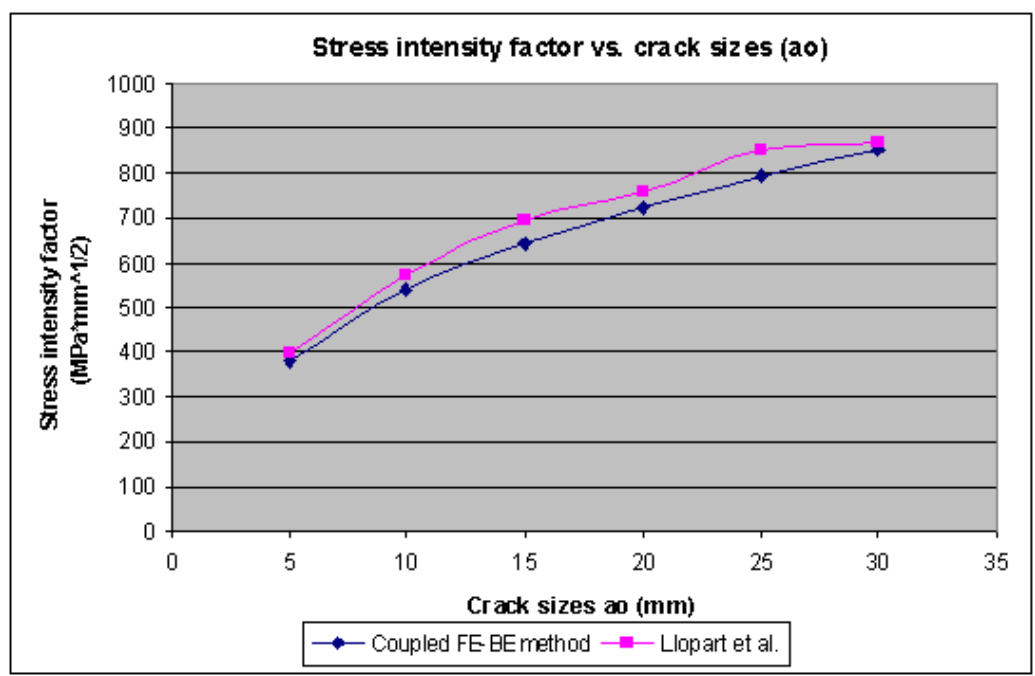

Figure 10: Stress intensity factors given by coupled FE-BE method and literature. 


\section{Summary}

The advantage of the coupled BE-FE method is obvious. If a proper re-analysis criterion is selected, the coupled method can avoid continuous remeshing of the sub-model after each increment which was employed in automatic finite element crack propagation method. Also, no rosette-like singular elements are needed in the analysis and for the same level of mesh complexity the stress intensity factor calculated with boundary element method is more accurate than finite element method. With coupled method, fatigue analysis using BE in the vicinity of the crack of a non-linear structure becomes easier. As the crack is only located in a local area, the general non-linear behaviour of the structure can still be captured by finite element method therefore it is possible to take advantage of both the non-linear capabilities of FE and the accuracy of fatigue analysis in BE.

\section{References}

[1] P. O. Bouchard, F. Bay, and Y. Chastel, Numerical modelling of crack propagation: automatic remeshing and comparison of different criteria. Computer Methods in Applied Mechanics and Engineering, 2003. 192: p. 3887-3908.

[2] P. O. Bouchard, et al., Crack propagation modelling using an advanced remeshing technique. Computer Methods in Applied Mechanics and Engineering, 2000. 189: p. 732-742.

[3] A. A. Becker, The Boundary Element Method in Engineering. 1992: McGraw-Hill Book Company.

[4] A. Portela, Dual Boundary Element Analysis of Crack Growth. 1993, Southampton, UK: Computational Mechanics Publications.

[5] M. D. Snyder and T. A. Cruse, Boundary-integral equation analysis of cracked anisotropic plates. International Journal of Fracture, 1975. 11(2): p. 315-328.

[6] T. A. Cruse, Two-dimensional BIE fracture mechanics analysis. Applied Mathematical Modelling, 1978. 2: p. 287-293.

[7] ABAQUS Analysis User's Manual. 2005, ABAQUS Inc.

[8] Ll. Llopart, et al., Investigation of fatigue crack growth and crack turning on integral stiffened structures under mode I loading. Engineering Fracture Mechanics, 2006(73): p. 2139-2152. 